\title{
Essais
}

ESSAIS

Revue interdisciplinaire d'Humanités

3 | 2013

Narration et lien social

\section{Les micro-récits d'une littérature-monde : Landolfi et Nabokov traducteurs de Pouchkine}

\section{Stanislas Gauthier}

\section{(2) OpenEdition}

12 Journals

Édition électronique

URL : https://journals.openedition.org/essais/9239

DOI : $10.4000 /$ essais. 9239

ISSN : 2276-0970

Éditeur

École doctorale Montaigne Humanités

\section{Édition imprimée}

Date de publication : 15 mai 2013

Pagination : 79-99

ISBN : 978-2-9544269-0-7

ISSN : 2417-4211

\section{Référence électronique}

Stanislas Gauthier, «Les micro-récits d'une littérature-monde : Landolfi et Nabokov traducteurs de Pouchkine », Essais [En ligne], 3 | 2013, mis en ligne le 01 septembre 2021, consulté le 08 septembre 2021. URL : http://journals.openedition.org/essais/9239; DOI : https://doi.org/10.4000/essais.9239 


\section{Les micro-récits d'une littérature- monde : Landolfi et Nabokov traducteurs de Pouchkine}

Stanislas Gauthier

Deux auteurs occidentaux de la même génération, Tommaso Landolfi (1901-1977) et Vladimir Nabokov (1899-1977), traduisent le poète russe Alexandre Sergueiévitch Pouchkine (1799-1837) après la Seconde Guerre mondiale. Pour Nabokov, émigré russe devenu américain en 1945, Pouchkine se confond avec la langue de l'enfance, perdue dans les soubresauts de la révolution d'octobre 1917. Landolfi, écrivain toscan peu connu en dehors de la péninsule italienne, fait entrer le russe dans un apprentissage des langues étrangères en grande partie autodidacte, qui passe à la fin des années 1920 par la soutenance de l'une des premières thèses italiennes en slavistique, sur Anna Akhmatova. Ni Landolfi, ni Nabokov, n'a fait mention de l'autre. Nous les rapprochons parce que tous deux ont travaillé la matière verbale, avant les idées évoquées par les mots, à une très petite échelle.

La démarche apparaît particulièrement surprenante quand ces deux écrivains traducteurs abordent Le Cavalier de bronze (Медный всадник, Miedny Vsadnik), un long poème narratif de Pouchkine. Écrit du 6 au 31 octobre 1833 à Boldino, ce texte qui raconte le parcours tragique d'un homme ordinaire confronté à la grande inondation du 7 novembre 1824 inspira de nombreuses lectures politiques, alors même qu'il fut considéré comme "le plus beau » ${ }^{1}$ poème de Pouchkine. Landolfi en publie une version intégrale (sans discours d'escorte) dans le premier volume de ses traductions ${ }^{2}$ chez l'éditeur Einaudi en 1961. En dépit de nombreux retards de publication dus aux politiques éditoriales, la traduction anglaise d'Eugène Onéguine par Nabokov paraît en 1964. D’abord établie à l'intention de ses étudiants, elle demeure indissociable d'un commentaire de plus de neuf cents pages qui replace les mots de Pouchkine dans une histoire

1 André Meynieux, "Avertissement ", in Pouchkine, Le Cavalier de bronze. Traduit sur les manuscrits originaux et publié par A. Meynieux, Paris, André Bonne, 1959, p. 5.

2 Aleksandr Puškin, Poemi e liriche, Torino, Einaudi, 1961.

3 Aleksander Pushkin, Eugene Onegin, Translation by V. Nabokov, New York, Bollingen, 1964. 
de leurs usages à travers la littérature et comprend de nombreux textes russes que Nabokov traduit lui-même. Un court extrait du Cavalier de bronze fait partie du florilège.

Notre hypothèse est que Landolfi et Nabokov se placent au plus près d'une naissance artistique. Ils tentent de retrouver la force d'ébranlement d'un classique enseigné dans les écoles soviétiques à l'issue d'une construction sociale qui valide son caractère national. Définis par André Akoun comme " ces formes de relations qui lient l'individu à des groupes sociaux et à la société, qui lui permet de se socialiser, de s'intégrer [...] et d'en tirer les éléments de son identité " ${ }^{4}$, les liens sociaux s'ancrent dans " des dynamiques affectives qui le $[s]$ portent ${ }^{5}$. Mais en privilégiant la sensualité du texte poétique, Landolfi et Nabokov limitent considérablement ces perspectives sociales, par ailleurs difficiles à retrouver dans la traduction de la poésie étrangère.

Ces questions de traduction entrent après la Seconde Guerre mondiale dans des lignes idéologiques qui imposent à la littérature de choisir son camp. Les relations internationales se tendent en une " opposition politique, stratégique, militaire autant que socio-économique, idéologique et culturelle [...] jouée entre deux blocs antagonistes structurés autour de deux superpuissances [les États-Unis capitalistes et l'URSS communiste] qui ne se sont jamais affrontées dans un conflit direct $»^{6}$. La Guerre froide est une période intéressante pour ce qui concerne la traduction en ce qu'elle met en lumière une incompatibilité entre ceux qui valorisent la fraternité entre les peuples au-delà du texte et d'autres qui considèrent la traduction avant tout comme un travail littéraire. René Nelli, traducteur des Cinq poèmes d'amour du poète catalan Jordi Sant Jordi en 1946, écrit que le poète "doit aux circonstances de nous émouvoir présentement comme s'il était le frère de tous les captifs d'aujourd'hui ». Citant ces propos, le poète communiste français Louis Aragon les commente ainsi : " un poème n'est pas écrit une fois pour toutes, [...] des circonstances imprévisibles pour l'auteur [...] vont s'emparer [de lui] plus tard en dehors de son auteur, et [...] le poème aura "changé de sens pour nous plaire" " 7 . Pour Aragon, la traduction, en profitant des analogies, doit promouvoir l'idée d'une entente internationale, ce qui dépasse les attentes de Landolfi et de Nabokov. Dans sa restitution d'une soirée organisée par une société savante de New York en 1940, le slaviste Edmund Wilson n'évoque que des " textes et des poèmes à la gloire et à la défense de Leningrad [qui]

4 André Akoun, "Lien social », in A. Akoun et P. Ansart, Dictionnaire de sociologie, Paris, Le Robert-Le Seuil, 1999, p. 307.

5 André Akoun, «Lien social », Dictionnaire de sociologie. Paris, Le Robert-Seuil, 1999, p. 308.

6 Marie-Pierre Rey, "La Guerre froide en questions », in Claude Quétel (dir.), Dictionnaire de la Guerre froide, Paris, Larousse, 2008, p. 11.

7 Louis Aragon, Chroniques de la pluie et du beau temps, précédé de Chroniques du Bel Canto, Paris, Les Éditeurs Français Réunis, 1979, p. 30-31. 
suivaient de toute évidence une "directive", car toutes les interventions des orateurs étaient presque toutes identiques [...] Tout le monde cita Mедный Всадник à qui mieux mieux, et parfois fort mal à propos $"^{8}$.

Les traductions de Nabokov et de Landolfi proposent des liens moins évidents entre les lecteurs. Nous souhaitons préciser dans cet article la nature sociale de ces liens. Pour répondre à cette problématique, nous nous intéresserons aux études de réception et de traduction dans leurs dimensions sociologiques et esthétiques, en une optique résolument comparative à la fois générale et inductive. Après avoir rappelé les usages sociopolitiques dont fait l'objet cette œuvre depuis son écriture, nous étudierons la singularité du lien social tissé par un récit poétique et nous comparerons les lectures à très petite échelle que Landolfi et Nabokov proposent du Cavalier de bronze.

\section{La fabrique du poète national}

Le poème narratif de Pouchkine est constitué de trois parties. Le prologue montre le tsar Pierre I $^{\text {er }}$ sur une plage déserte, imaginant les beautés de la ville de Saint-Pétersbourg. Les deux parties du récit qui suit se déroulent cent ans plus tard, alors que la ville est déjà construite. Eugène, un noble déclassé qui rêve d'épouser Paracha et de fonder une famille, se trouve pris dans la grande inondation de novembre 1824 et échappe de justesse à la mort. Seule la statue de Pierre ${ }^{\text {er }}$, chef-d'œuvre du Français Falconet, place du Sénat, résiste à la tempête. La seconde partie montre le retour à la vie ordinaire. Eugène se met à la recherche de Paracha. Quand il découvre qu'il ne reste rien de la maison qui l'abritait, le jeune homme sombre dans la folie. Après sans doute une longue errance, Eugène se retrouve de nouveau près de la statue du tsar Pierre ${ }^{\text {er. }}$. Dans un geste de défiance, il menace du poing l'empereur. La statue

8 Lettre du 7 mars 1943 de Edmund Wilson à Vladimir Nabokov, in V. Nabokov, E. Wilson Correspondance 1940-1971, Traduit de l'anglais par Christine Raguet-Bouvard. Paris, Rivages, 1979, p. 109.

On ne relève que deux traductions en italien du Cavalier de bronze avant 1961. La première est issue d'une réimpression en 1937 d'un recueil signé de la duchesse d'Andria qui comprend également Boris Godunov. La seconde en 1940 est due à Giovanni Gandolfi. En comparaison, on compte huit traductions du Cavalier de bronze en anglais. Quelques-unes paraissent en revue (celle d'Oliver Elton en 1934, d'Edmund Wilson en 1938) pour ensuite être insérées dans une anthologie de poésies russes. Le plus souvent cependant, parait directement un volume comprenant des poésies de différents auteurs russes. Charles Fillingham Coxwell en 1929, Bayard Simmons en 1944, John Fennell en 1964 font paraitre ainsi leurs anthologies. Parfois les poèmes du seul Pouchkine, après une publication en un recueil hétérogène, sont regroupés. Charles Turner publie un volume de ce genre en 1899. Enfin, seul le prologue a été publié par Nadine Jarintzov en 1917 et par Dimitry Obolensky en 1962. Voir Claudia Scandura, Letteratura russa in Italia. Un secolo di traduzioni. Rome, Bulzoni Editore, 2001, et Lauren G. Leighton, A Bibliography of Alexander Pushkin in English. Studies and Translations, Lewiston, The Edwin Mellen Press, Studies, in Slavic Languages and Literature, vol. 12, 1999. 
descend alors de son socle et poursuit le jeune homme jusqu'à l'épuisement. On retrouve le corps d'Eugène sur une plage déserte, étendu sur le seuil d'une maison que la mer a rejetée et qui pourrait être celle de Paracha.

Depuis son écriture, le texte de Pouchkine a régulièrement fait l'objet de lectures sociopolitiques. Du vivant du poète, le tsar Nicolas ${ }^{\text {er }}$ qui s'est désigné son censeur personnel refuse la publication du texte, car il a bien perçu la charge polémique qui associe le cataclysme de 1824 à la place du Sénat. Inspirés par la Révolution française et par la monarchie constitutionnelle anglaise, les jeunes aristocrates à la tête des armées ont organisé une mutinerie sur cette même place le 14 décembre 1825, le jour du sacre de Nicolas ${ }^{\text {er }}$. Ce qui devait aboutir à un régime libéral ne conduisit qu'à l'exécution des meneurs décembristes et à de nombreuses déportations en Sibérie. Le Cavalier de bronze évoque cet échec russe.

Dans l'un de ses articles intitulé " De la narodnost' en littérature ", écrit entre 1825 et 1826, le poète russe évoque l'émergence d'un caractère national incompréhensible aux étrangers : "La narodnost' chez un écrivain est une qualité qui ne peut être appréciée à sa juste valeur que par ses compatriotes, pour les autres elle n'existe pas, ou bien peut au contraire être perçue comme un défaut. Le savant allemand s'indigne de la courtoisie des héros de Racine, le Français rit de voir Coriolan provoquer son ennemi en duel chez Calderón "?. Dans le contexte des revendications nationales liées au romantisme, Pouchkine déclare incompréhensible à des yeux étrangers le lien qui relie un peuple à sa littérature. Cependant, Le Cavalier de bronze entre en résonance avec l'œuvre du poète polonais Mickiewicz. À l'heure de la révolte de la Pologne contre Nicolas Ir dans les années 1830-1831, Mickiewicz s'en prend violemment à la servilité du peuple russe. Pouchkine répond en prenant fait et cause pour son pays. Mickiewicz fait alors paraître Oleszkiewicz, un poème à charge qui dépeint la ville de Pierre comme une nouvelle Babylone. Pierre I ${ }^{\text {er }}$, dans la statue de Falconet, ne ferait pas un signe d'apaisement mais de domination. Pouchkine tient compte de ces critiques dans Le Cavalier de bronze.

Par ailleurs, le poète n'a pas gardé la même popularité toute sa vie. À partir de 1827, les lecteurs russes regrettent ses poèmes narratifs pittoresques et l'œuvre seule ne peut expliquer le caractère national que l'on vante souvent. Associant le parcours artistique et le mythe individuel, Gogol, dans son article "Quelques mots sur Pouchkine ", recueilli dans Arabesques en 1834, qualifie le poète d' "entièrement russe " et lui prête la modestie face au poids du destin (смирение), encore déclinée en 1837 par Lermontov dans " La mort du poète $»^{10}$. Très rapidement, on emploie à propos de Pouchkine après 1837 un

9 Traduit par Catherine Lemagnen, «Le concept de narodnost' dans la pensée russe du XIX e siècle », in « Monde russe et identités. » La Revue russe 36, Paris, Institut des études slaves, 2011, p. 11-20.

10 Mikhaïl Lermontov, CEuvres poétiques, Lausanne, L’Âge d'homme, 1985, p. 79-82. 
trope hagiographique ${ }^{11}$, « le soleil de notre poésie » (солнце нашей поэзии), qui évoque les mots utilisés pour désigner les princes martyrs de Russie. Entrant dans un récit national, le poète est confondu avec les figures de Gleb et de Boris, les deux saints protecteurs qui voulurent éviter une lutte fratricide à la Russie kiévienne en abandonnant toute résistance face aux attaques de leur frère Sviatopolk. Selon Paul Debreczeny,

à l'époque de Pouchkine, [...] les princes et les tsars avaient perdu leur capacité de représenter la nation. Avec la publication de la sixième lettre philosophique de Pietr Tchaadaev accusant la société russe à une grande échelle, avec la quête des Slavophiles d'une identité nationale et avec le rejet par le mouvement radical naissant du passé tourmenté par la tyrannie, la voie était libre pour un nouveau genre de prince - un prince culturel - pour représenter la nation. Ayant exprimé l'esprit du peuple à travers sa poésie divinement inspirée, et ayant été soumis au martyre, Pouchkine pouvait intercéder avec l'Esprit Universel des philosophes romantiques, assurant un rôle honorable pour les Russes dans la marche en avant de l'histoire du monde. ${ }^{12}$

Lors de l'inauguration de la statue du poète réalisée par Alexandre Opekushin à Moscou en 1880, le discours de Dostoïevski rassemble toutes ces images - la résignation gogolienne, la figure de martyr, le caractère protecteur - et consacre définitivement Pouchkine en poète national russe. D’après Clyde Kluckhohn, les mythes et les rituels religieux " promeuvent la solidarité sociale, augmentent l'intégration de la société en fournissant une formulation précise de ses attitudes morales ultimes ${ }^{13}$. Sa situation de poète trahi par l'Empereur et par la Cour, sa mort en duel contre un étranger sont des éléments de l'histoire de Pouchkine qui devaient s'avérer particulièrement efficaces pour la promotion d'une image de poète populaire, humble parmi les humbles de la nation. Au tournant du siècle, lors du centenaire de la naissance du poète, des anthologies furent publiées en grand nombre. On profita

de l'anniversaire pour diffuser [également sous le nom de Pouchkine] une large gamme de produits comprenant des cigarettes, des allumettes, des sucreries, des stylos, de la papeterie, de l'encre, de la liqueur, des couteaux, des montres, des vases, des coupes, des chaussures, des habits, des lampes, des éventails et du parfum ("Bouquet Pouchkine»), un ensemble de portraits et de cartes postales, un jeu de société ( le Duel de Pouchkine»). ${ }^{14}$

Tout en prenant une dimension religieuse, l'image du poète a été popularisée par des voies profanes, ce qui lui permit d'être connu parmi les sphères les plus diverses de la population. Un lecteur étranger à la culture russe imagine diffici-

11 Voir Paul Debreczeny, Social Functions of Literature. Alexander Pushkin and Russian Culture, Stanford, Stanford University Press, 1997, p. 224.

12 Paul Debreczeny, ibid., p. 224. Notre traduction.

13 Clyde Kluckhohn, "Myths and Rituals : A General Theory ", The Harvard Theological Review, vol. 35, n 1 (janvier 1942), p. 65. Notre traduction.

14 P. Debreczeny, op. cit., p. 229-230. Notre traduction. 
lement une telle construction. Profitant d'un kä̈ros, moment singulier de l'histoire nationale, le poète national exprime d'une manière inouïe la réalité russe. Il renouvelle ses lieux communs (Saint-Pétersbourg, Pierre le Grand) et relie le parcours exemplaire de son personnage à celui d'autres Pétersbourgeois. L'histoire se déroule dans le quotidien le plus familier et parvient à exprimer la dureté de la vie de nombreux lecteurs russes. Il trouve également une forme épique qui évite toute conclusion idéologique et ruine en cela toute tentative de clarification qui voudrait épuiser la pluralité des sens. " [I]nstrument pour penser le conflit en profondeur et donner voix à tous, vaincus et vainqueurs $»^{15}$, l'œuvre du poète national synthétise ainsi le caractère russe tout en en révélant la richesse.

La réception du poète fut cependant peu uniforme. Le mouvement radical des années 1850 à 1870 le considère moins utile qu'une paire de bottes. Remis à l'honneur à la fin du XIXe siècle par les symbolistes, Pouchkine est de nouveau refusé par les Futuristes à partir de 1910, puis par les bolchéviques en 1917, puisque "le premier mouvement fut de détruire toute la culture ancienne comme un attribut du tsarisme $»^{16}$. En termes de temps disponible et d'intensité émotionnelle, une concurrence existait entre la lecture de Pouchkine et l'idéologie communiste qui se diffusait grâce à des lieux de socialisation multiples, comme les cellules du parti et ses organisations de jeunesse. Mais dans les goûts que revendiquait Lénine, Pouchkine se trouvait en bonne place, ce qui légitimait l'intérêt qu'on lui portait. Alors même que le régime stalinien tente de simplifier considérablement la dimension idéologique du poète dans les commémorations de 1937 et de 1949, ces journées ne ressemblent pas exactement aux autres rituels soviétiques, si l'on en croit Paul Debreczeny : "l'occasion est la plus confuse - officiellement permise, cependant pleine de spontanéité interdite $»^{17}$.

« [E]n 1933, l'unification des manuels est décidée, ce qui permet d'approvisionner toutes les écoles et supprime toute rivalité théorique $»^{18}$. Le régime soviétique a accepté peu à peu l'héritage impérial, et l'historienne Anna Pankratova a été chargée de réécrire tous les manuels d'histoire en ce sens. Du début des années 1920 à 1935, l'idéologie officielle suivait les thèses de l'historien Mikhail Pokrovski qui résumait l'histoire russe à une suite de jacqueries aboutissant de manière nécessaire à la création des soviets. Les tsars n'avaient joué aucun rôle dans la construction du pays. Pierre le Grand était au service du « capitalisme marchand ». "Après l'écrasement de l'école sociologisante de Pokrovski, [...] on avait assisté à la résurrection du passé national, par tranches successives

15 Voir Florence Goyet «L'Épopée », p. 6 [en ligne] : http://www.vox-poetica.com/sflgc/biblio/ goyet.html (page consultée le 28 mars 2013). Nous ne pouvons développer la dimension épique, compte tenu de l'espace qui nous est accordé.

16 P. Debreczeny, op. cit., p. 231. Notre traduction.

17 P. Debreczeny, op. cit., p. 237. Notre traduction.

18 Gaston Mialeret et Jean Vial, Histoire mondiale de l'éducation, t. 3 de 1815 à 1945, Paris, PUF, 1981, p. 130. 
[...] : Pierre le Grand d'abord, puis Ivan IV, dit le Terrible, Alexandre Nevski, Dimitri Donskoö (tous deux canonisés par l'Église) [...] $»^{19}$. En somme, Pouchkine faisait partie du même panthéon dans l'avènement de l'URSS.

Le contexte de la Guerre froide se situe dans la droite ligne de la suspicion à l'égard de l'étranger que connaît le régime bolchévique depuis son origine. Dans cette perspective, la littérature comme la traduction doivent transmettre la nécessité de liens sociaux internationaux entre les masses populaires et véhiculer une image positive du régime soviétique. S’inscrivant dans la lignée de Bielinski qui voyait dans Le Cavalier de bronze « la lutte entre la volonté collective, la nécessité historique représentées par Pierre et la volonté individuelle représentée par Eugène $~^{20}$, la critique soviétique a relu la révolte de l'homme de rien contre le tsar en prenant le parti de Pierre ${ }^{\text {er }}$ " représentant de la nécessité historique et de la raison d'État, au regard desquelles est négligeable le destin individuel d'un quelconque Eugène, victime nécessaire, inévitable $»^{21}$. Le scandale provoqué par l'ouvrage d'André Siniavski, qui écrivait en 1975 que «Pouchkine fut prodigue de petits riens » ou encore qu'il « érige[a] le bavardage en vrai principe de style ${ }^{22}$, montre la rareté des lectures non idéologiques dans le monde russe.

Trois conclusions peuvent être tirées de ce rapide panorama de la réception russe de Pouchkine. Le poète russe ne fut pas oublié, car ses œuvres se caractérisaient par leur saturation symbolique, par une concentration rigoureuse du récit et par leur éclat métaphysique ${ }^{23}$. Elles firent l'objet, dès l'origine, d'une dramatisation qui leur permit de constituer un patrimoine national russe et d'accompagner la formation de liens sociaux. Cependant le texte, utilisé à des fins politiques, a été simplifié pour permettre une traduction plus efficace.

\section{La « société secrète ${ }^{24}$ des lecteurs}

Face à cette primauté du sociopolitique, Landolfi et Nabokov défendent chacun de leur côté une autonomie de la littérature, indissociable selon eux de l'autonomie de l'individu dans la société. Le refus bien connu chez Nabokov d' " une approche socioéconomique ${ }^{25}$ peut s'expliquer entre autres par le choix

19 Georges Nivat, "Un styliste de l'histoire", in Vers la fin du mythe russe. Essais sur la culture russe de Gogol à nos jours, Lausanne, L'Âge d'homme, 1983, p. 162-165.

20 A. Meynieux, Le Cavalier de bronze, op. cit., p. 11.

21 A Meynieux, Le Cavalier de bronze, op. cit., p. 12.

22 André Siniavski, Promenades avec Pouchkine, traduit du russe par Louis Martinez, Paris, Éditions du Seuil, 1975, p. 15 et p. 75.

23 Philippe Sellier, «Qu’est-ce qu’un mythe littéraire ?», in Littérature, vol. 55, n 55, p. 112-126.

24 Julien Gracq, La littérature à l'estomac, Paris, José Corti, 1950, p. 22.

25 Lettre à Vaun Gillmor du 26 juin 1957, in V. Nabokov, Lettres choisies 1940-1977, traduction de l'anglais de Christine Bouvart, Paris, Gallimard, 1989, p. 282. Voir par ailleurs l'interview 
d'une esthétique du détail singulier qui refuse de rompre la magie de la fiction pour la lire à la lampe de théories générales. Dans Des Mois, le troisième tome paru en 1967 de carnets autofictionnels qui présentent de multiples narrations, Landolfi évoque l'anarchie que l'individu est susceptible de provoquer :

On pourrait objecter aussi que le système social est justement le système du plus grand nombre; mais dans ce cas, qu'au moins on ne le fasse pas passer pour un système, puisqu'une seule exception (une seule volonté contraire, en l'occurrence) fait exploser n'importe quel système de l'intérieur.

[...] D'où découlent, plus ouvertement, l'impossibilité d'un système politicosocial, et l'une de mes idées de socialité partielle : chacun a le devoir d'exercer une action bénéfique dans son domaine personnel, et celui de ne pas aller au-delà, en refusant toute idéologie, ou disons plutôt tout mensonge. ${ }^{26}$

Ni Landolfi, ni Nabokov ne valorisent malgré tout la communauté. ${ }^{27}$ La place de Nabokov parmi l'émigration n'est d'ailleurs pas simple à définir. Après la révolution d'octobre 1917, les émigrés se sont vus comme les représentants de la vraie Russie, les dépositaires de l'héritage culturel et spirituel refusé par les bolchéviques. Les commémorations dans le monde entier lors du centenaire de la mort de Pouchkine en 1937 ont permis aux émigrés de se reconnaître et de défendre la Russie en exil. Peu à peu l'Église a joué un rôle grandissant dans le renforcement du lien social qui s'établissait entre les diffé-

parue dans Playboy en janvier 1964, reprise dans V. Nabokov, Partis pris, Paris, Éditions 10/18, 2001, p. 28-55.

26 T. Landolfi, Des Mois, traduction de l'italien de Monique Baccelli, Paris, Éditions Allia, 1995, p. 81.

27 En Allemagne, Ferdinand Tönnies (1855-1936) a défini les associations entre les volontés humaines selon le mode de la communauté (Gemeinschaft) "vie réelle et organique ", ou de la société (Gesellschaft), " construction idéelle et mécanique " (Voir Ferdinand Tönnies, Communauté et Société, Paris, PUF, 2010, p. 13). Selon l'anthropologue Stéphane Vibert, d'autres concepts doivent être utilisés pour approcher une culture "à prééminence holiste " comme la Russie. « La définition d'une narodnost' (esprit national) justifiant la mission de la Russie dans l'histoire suppose une tension entre particulier et universel résolue par l'appel à un mode spécifique de communauté (sobornost', église "communion des saints" légitimant le mir comme corps social) et d'individu (défini par la tselnost "intégrite', restaurée par une foi subordonnant le rationalisme) [...] la récusation d'un pouvoir de médiation sacrale (contre la trahison "occidentale" de l'empire pétersbourgeois) instaure une entité collective mystique (le peuple russe) au fondement d'une culture à la fois "orthodoxe" et "universelle", intégrant les deux sens du mot pravda: une "vérité" donnée incarnée dans une "justice" effectivement vécue sur un plan sociocommunautaire. " Voir Stéphane Vibert, "La quête russe de l'universel : mouvement slavophile et hiérarchie de valeurs socio-communautaires (1825-1855) ", in Revue des études slaves, t. 73, fascicule 2-3, 2001, p. 519-530. Selon Georges Nivat, le terme de " [...] sobornost, ou conciliarité, ou "penser ensemble", un terme venu de la théologie, [...] qui distingue la pensée individuelle dans l'acuméné ecclésiale de la pensée individuelle dans le libéralisme occidental : l'individualisme dans l'unanimité est une tendance de la pensée russe qui sévit régulièrement. » Voir Georges Nivat, Transitions. «Bilan de la culture soviétique ». Édité par G. Nivat, vol. XLI, Bruxelles, Critème/ Ieug, 2002, p. 16. 
rents membres de la diaspora ${ }^{28}$. Cosmopolite dès son enfance, peu religieux, Nabokov n'est absolument pas indifférent à la culture russe, mais il y opère des choix. Il ne suit ni Georgi Adamovitch, pour qui l'œuvre de Pouchkine appartient au passé, ni Dimitri Merejkovski qui lisait dans le Cavalier de bronze "le conflit entre la Chrétienté et le paganisme, les deux forces premières en lutte dans la civilisation européenne ${ }^{29}$. Pour Nabokov comme pour Khodassevitch, si l'émigration n'a pas su écrire une ouvre assez forte, c'est bien qu'elle n'a pas su transcrire le tragique humain (c'est-à-dire le caractère universel à travers le récit d'un destin) qui l'accompagnait.

Grand lecteur pendant son adolescence de la poésie de Blok inspirée de chants populaires, Nabokov est aussi le traducteur de Слово о полку Игореве (Slovo o polku Igorevye) un poème épique du XII e siècle, dont le slaviste français André Mazon a mis en doute l'authenticité ${ }^{30}$ pendant les années 1930 ainsi que dans un livre paru en 1940, Le Slovo d'Igor. En découlent de violentes controverses avec Roman Jakobson qui, émigré mais investi d'un devoir patriotique, répond en 1948 dans La geste du Prince Igor'. Épopée russe du douzième siècle. Cette insertion des textes dans des questions de politique nationale a entraîné chez Nabokov des changements radicaux dans sa façon de considérer la traduction. Dépassant le point de vue de Mazon qui soutient l'idée d'un pastiche $\mathrm{du}$ XVIII siècle de la Zadonshchina, un autre poème épique de la fin du XIV ${ }^{e}$ siècle, refusant d'en faire avec Jakobson un exemple de la spécificité de la Russie kiévienne contre le reste de l'Europe du XII ${ }^{e}$ siècle, Nabokov insiste sur la singularité de l'œuvre littéraire dans le contexte religieux de la Russie médiévale et souligne sa dimension artistique. Il met en exergue le genre poétique en traduisant le titre par The Song of Igor's Campaign (La Chanson de la campagne d'Igor) et défend l'idée d'une ouvre qui dépasse son lieu de naissance, encore lisible même aux États-Unis. Mais parce qu'il défend la variété et la richesse de la culture russe, Nabokov ne remet en question ni la langue, ni le contexte historique qui caractérisent le texte. Quand il écrit qu' " à travers le brouillard des sagas scandinaves certains ponts ou ruines de ponts peuvent être distingués liant les romances d'Écosse et du Pays de Galles avec celles de Kiev »" , il perçoit des similitudes. Cette vision de la traduction met en récit des analogies, mais souligne également la disparition et la difficulté des liens interculturels. Des implications politiques certaines en découlent puisque Nabokov renvoie dos à dos les partisans d'un monde sans différences et les nationalistes.

28 Georges Nivat, "La Treizième tribu ", in Russie-Europe. La Fin du schisme. Études littéraires et politiques, Lausanne, L'Âge d'homme, 1993, p. 653.

29 A. Meynieux, Le Cavalier de bronze, op. cit., p. 11.

30 Voir Iakov Gordine, «L'Histoire comme acte d'opposition », in Transitions. « Bilan de la culture soviétique ", édité par G. Nivat, op. cit., p. 61-74.

31 V. Nabokov, Song of Igor's Campaign, New York, Ardis, 1960 (1988 pour l'édition consultée), p. 15. Notre traduction. 
Le travail de Jakobson a été commenté par Landolfi dans l'un de ses articles pour " Mondo » le 30 mars 1954 lors de la publication de la traduction italienne du texte par le slaviste italien qui émigrera aux États-Unis, Renato Poggioli. Après avoir mentionné les doutes sur l'authenticité du Slovo, Landolfi écrit que :

De ceux-là semble avoir triomphé il y a peu de manière lumineuse Jakobson justement (défini par Poggioli "prince des linguistes et des slavistes contemporains"), c'est-à-dire le même à qui on doit la présente édition critique extrêmement valable. De façon que rien désormais ne s'oppose à ce que nous nous abandonnions sans soupçon à cette lecture. ${ }^{32}$

Landolfi est un grand admirateur de Khlebnikov - «le plus grand poète $»^{33} \mathrm{du}$ $\mathrm{XX}^{\mathrm{e}}$ siècle, selon Jakobson - dont il traduit deux poèmes pour la revue L'Italia Letteraria le 19 janvier 1935. Le russe demeure pour lui une source de désir et de dépaysement au sens fort. Les contraintes imposées par le régime soviétique ne lui ont pas rendu insupportables les déclarations à l'emporte-pièce des avant-gardes. Cherchant une synthèse entre les conteurs russes et français du XIX ${ }^{\mathrm{e}}$ siècle, le romantisme allemand et les poètes soviétiques, l'écrivain italien vise, au-delà du récit, une utopie verbale qui ne maintient pas forcément le lien avec le lecteur. À l'horizon apparait une direction poétique vers laquelle semble tendre tout l'œuvre de Landolfi. Carlo Bo indique que «tout [son] romantisme [...] naît de ce choix de se mettre à part, de vivre en dehors de la société, d'exalter son "exclusion". [...] [La] chose dans l'absolu la plus importante pour lui était de laisser aux hommes toute la liberté possible, de ne pas les illusionner, de ne pas les tromper $»^{34}$. Cette défense de la liberté n'encourage pas l'individualisme sociologique, dans la mesure où Landolfi et Nabokov placent leurs personnages dans des alternatives morales qui ne peuvent laisser indifférent. En s'adressant d'abord au lecteur isolé plutôt qu'aux communautés, ils donnent à ce dernier la possibilité de penser ses contraintes et de se poser lui-même ses limites.

On peut expliquer ce choix en revenant à l'espace littéraire italien, qui en raison de l'imitation de Dante, Boccace et Pétrarque, "ne s'est constitué que fort tard $"^{35}$, selon Pascale Casanova. Après les chiffres impressionnants des traductions sous le fascisme ${ }^{36}$ dus à la méconnaissance par le régime de

32 Voir T. Landolfi, "Un antico canto epico ", in Gogol' a Roma, Milan, Adelphi Edizioni, 2002, p. 63. Notre traduction.

33 Roman Jakobson, Russie, folie, poésie, Paris, Seuil, 1986, p. 25.

34 T. Landolfi et Idolina Landolfi, Euuvres complètes, t. I, Milan, Rizzoli, 1991, p. IX. Notre traduction.

35 Pascale Casanova, La République mondiale des Lettres. Paris, Éditions du Seuil, 1998-2008, p. 91-92.

36 Selon Christopher Rundle, l'Italie a publié plus de traductions qu'aucun autre pays au monde pendant les années 1930. Les traductions n’y ont d'ailleurs jamais été traitées différemment des autres publications. Avant la loi du 18 janvier 1943 qui obligeait les éditeurs à demander l'autorisation d'imprimer, le régime intervenait peu parce que l'autocensure était générale et conduisait à 
Mussolini de l'ouverture qu'elles représentaient, "la littérature russe ", dans le contexte italien de l'après-Seconde Guerre mondiale, " a été identifiée avec l'Union soviétique. [...] [D]ans le choix des textes à traduire, on préfere accentuer le caractère sociopolitique $\aleph^{37}$.

L'impegno civile fut au centre de la poétique des années qui vont de l'aprèsguerre à 1975. [...] D'une part on avait fait le procès à l'hermétisme florentin d'avoir pactisé avec le fascisme, ou, en tout cas, de ne pas avoir su le dénoncer, par indifférence, ou par lâcheté, mais aussi en fonction de la conviction que la poésie peut s'écrire et se développer sans tenir compte de la puanteur ambiante. Après la guerre, et dans un mouvement critique, la poésie s'est interrogée sur la nécessité de son "impegno civile ».

D’autre part, cet impegno politique, né de la résistance, a engagé la poésie italienne " à gauche ", selon une orientation qui était globalement celle du parti communiste $[\ldots] .{ }^{38}$

Peu visible jusqu'au milieu des années 1950 en raison du maccarthysme, la littérature engagée aux États-Unis a par la suite porté ses critiques sur les tabous de la société américaine ainsi que sur la politique extérieure du pays. Quand elle a tenté de promouvoir certaines œuvres artistiques, un véritable regard critique lui fit défaut. Les anthologies consacrées à la littérature russe rassemblèrent auteurs soviétiques et classiques du XIX ${ }^{\mathrm{e}}$ siècle sans distinguer les textes selon leur valeur. La décision de Nabokov de publier en 1964 Eugène Onéguine en trois volumes - une traduction accompagnée de deux volumes de notes - fait de l'œuvre de Pouchkine un massif impossible à ignorer de la culture russe, en même temps qu'elle raconte un poète ouvert aux autres littératures.

\section{Les petites narrations et le partage du sensible}

Les difficultés de la traduction du Cavalier de bronze résident dans l'extrême concision de la langue de Pouchkine qui comporte très peu d'adjectifs. Le mot acquiert tout son volume au prix d'un travail sur les sonorités extrêmement poussé. Comme l'explique Boris Uspenskij, Pouchkine se situe dans une situation intermédiaire où les différents registres stylistiques ne sont pas encore " unifiés ». La " couleur » d'un mot (slavon, populaire, étranger entre autres) est encore ressentie de façon extrêmement vive par le lecteur ${ }^{39}$.

une importante expurgation des livres. Voir Christopher Rundle, « Fascist Italy », in Derek Jones, Censorship : a world encyclopedia. Londres, Fitzroy Dearborn, 2001, p. 2444-2446.

37 C. Scandura, Letteratura russa in Italia. Un secolo di traduzioni, op. cit., p. 21. Notre traduction.

38 Martin Rueff, «Entretien avec Jean-Patrice Courtois ", Le nouveau recueil, n 81, Paris, Champ Vallon, décembre 2006-février 2007, p. 78.

39 Voir Boris A. Uspenskij, Storia della lingua letteraria russa. Dall'antica Rus' a Puskin. Edizione a cura di Nicoletta Marcialis, Roma, Il Mulino, 1993, p. 203. 
Après que le fascisme avait interdit l'usage des langues vernaculaires, alors que l'américanisation uniformisait l'italien (ce dont se plaindra plus tard un Pasolini), Landolfi reproduit les différences de registres dans sa traduction des pensées d'Eugène. Ses vers laissent alors de côté la répartition des rimes ${ }^{40}$.

\section{Traduction de Landolfi}

"Sposarsi? io? e perché no?

È un peso anche, s'intende;

$\mathrm{Ma}$ che, son giovane e robusto,

Son pronto a darmi attorno giorno e notte;

In qualche modo mi metterò su

Umile e semplice un rifugio,

E lì sistemerò Parascia.

Passerà forse un anno o due -

Otterrò un posticino, ed a Parascia

Confiderò la nostra famigliuola

Nonché l'educazione dei figliuoli...

E vivremo, e così fino alla tomba

La mano nella mano, noi due procederemo,

E i nipoti ci seppelliranno... ».

\section{Mot à mot en français de cette traduction ${ }^{41}$}

"Me marier? Moi ? et pourquoi pas ?

C'est un poids aussi, ça s'entend ;

Mais quoi, j'suis jeune et robuste,

J'suis prêt à en abattre autour, jour et nuit ;

De toute façon, je me mettrai sous

Un abri humble et simple,

Et je l'installerai, Paracha.

Il passera peut-être un an ou deux -

J'obtiendrai un plaçou, et à Paracha

Je confierai notre petite famille

Ainsi que l'éducation des petitous...

Et nous vivrons, et comme ça, jusqu'à la tombe

Main dans la main, nous cheminerons à deux,

Et les petits-enfants nous enterreront...".

\section{Texte original ${ }^{42}$}

« Жениться? Мне? зачем же нет?

Оно и тяжело, конечно;

Но что ж, я молод и здоров,

Трудиться день и ночь готов;

Уж кое-как себе устрою

40 Jacques Derrida a montré de manière décisive dans "Qu'est-ce qu’une traduction relevante? » que les traductions qui se disent les plus « fidèles » s'élaborent elles aussi à partir de choix. Voir Quinzièmes Assises de la traduction littéraire, Arles, Actes Sud, 1999.

41 Notre traduction. Nous n'avons pas tenté de restituer le schéma métrique.

42 Александр Пушкин, Полное собрание сочинений в одном томе, Moscou, Издательство Альфа-Книга, 2010, р. 619. 


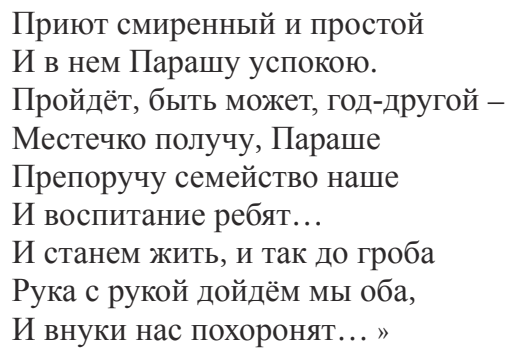

Le vocabulaire employé ici fait contraste avec les formules majestueuses qui présentaient le tsar Pierre dans le prologue du poème. "Comparé à la conduite, fluide et musicale de Lo Gatto, le vers de Landolfi donne un résultat plus âpre, et pas seulement à cause de l'absence de rimes. Même si l'apocope des verbes est habituellement évitée, le même registre linguistique apparaitt plus archaïque, plus courant dans le vocabulaire recherché ou désuet, quelquefois dans le toscanisme ${ }^{43}$. La traduction ne cherche pas l'adhésion du lecteur, mais au contraire son attention.

Dans sa traduction d'Eugène Onéguine, Nabokov ne traduit quant à lui que onze vers et ne donne l'original russe que pour un seul. L'ouvrage présente une ambition politique, dans la mesure où une œuvre qui passe pour nationale (Le Cavalier de bronze) s'insère dans un imaginaire plus large (auquel se rattache Onéguine) constitué de lectures occidentales. La traduction en " anglais rocailleux ", selon les propres mots de Nabokov, rompt le pacte de communication habituellement attendu dans ce genre d'exercice. Le commentaire devient un dictionnaire culturel où de nombreuses digressions malicieuses précisent le sens des mots. Refusant la connivence avec ses lecteurs, Nabokov en reste à une analyse des procédés littéraires. Les rapports avec la société ne sont cependant pas complètement oubliés. Si la fragilité du lien interpersonnel qui relie Eugène à l'espace social pétersbourgeois n'est pas évoqué, Nabokov évoque le manque de solidarité des autres citadins, ainsi que " la révolte d'un fou ", «la seule concevable, en Russie, de la part d'un simple sujet, contre un pouvoir tout puissant $"^{44}$. Mais il le fait sous un angle littéraire, en rappelant que Pouchkine

43 Michele Colucci, "Le traduzioni italiane del Novecento di poesia puškiniana ", in Paola Buoncristiano, Puškin, la sua epoca e l'Italia. Soveria Mannelli, Rubbettino, 2001, p. 109. Notre traduction.

44 A. Meynieux, Le Cavalier de bronze, op. cit., p. 10. Pétri de la morale de la III ${ }^{e}$ République française, Émile Durkheim affirmait par exemple que le divorce et les conjugalités non institutionnalisées comportaient des risques d'anomie, autrement dit de dérégulation sociale. Il préconisait notamment le mariage comme un rempart contre le suicide. Voir notamment Émile Durkheim, Le Suicide. Étude de sociologie. Paris, PUF, 1930. On trouve ainsi pages 428-429 : «Par conséquent, la seule façon de remédier au mal, est de rendre aux groupes sociaux assez de consistance pour qu'ils tiennent plus fermement l'individu et que lui-même tienne à eux. Il faut qu'il se sente davantage solidaire d'un être collectif qui l'ait précédé dans le temps, qui lui survive et qui le déborde de tous côtés. À cette condition, il cessera de chercher 
donne dans son œuvre le même nom à des individus séparés par la violence des circonstances. Le personnage principal d'Onéguine comme celui du Cavalier de bronze s'appelle Eugène.

Onéguine partit hiberner juste avant la calamiteuse inondation du 7 novembre 1824 (après laquelle les coûteuses festivités sociales comme celles au cours desquelles il a pu voir Tatiana, étaient temporairement interdites par un décret du gouvernement). En d'autres termes, Pouchkine, très avantageusement pour la structure du roman, fait dormir Eugène durant le désastre. Un autre Eugène, cependant, est en train de perdre pendant ce temps sa fiancée dans les eaux en furie, et est en train de devenir fou par l'imagination d'un galop d'une statue équestre dans le poème que Pouchkine a consacré à cette crue, Le Cavalier de bronze (composé en 1833). La manière dont Eugène Onéguine, dans son hibernation, prête son prénom à cet homme malheureux est très amusant [...] Le Cavalier de bronze est aussi lié à E[ugène] O[néguine] par une remarquable série de strophes dans la séquence rythmique d'EO, Le Pedigree de mon héros (Rodoslovnaya moego geroya 1832) ; Pouchkine a hésité sur celui des deux Eugène auquel elle devait s'appliquer, puis choisit un héros tout à fait autre (Ivan Ezerski). [...] $]^{45}$

Plus que les récits religieux, anthropologiques ou politiques, c'est le récit littéraire qui permet à Nabokov de penser la réalité. La très grande distance qu’adopte ici le commentateur souligne l'isolement radical des individus et rappelle la difficulté des liens sociaux.

Quand il commente la nuit pétersbourgeoise évoquée à la strophe $43 \mathrm{du}$ premier chapitre d'Onéguine, Nabokov évoque le face à face d'Eugène et de Pierre le Grand, mort un siècle plus tôt, sur le mode fantastique d'une rencontre de sonorités.

4 / sur le pavé pétersbourgeois / Po Peterburgskoy mostovoy : prononcé pa peterbûrskoy mastovôy. Notez la double allure dans cette ligne et la frappante répétition $p a$ pe avec laquelle on attaque le vers. Pouchkine employa le même procédé dans son long poème Le Cavalier de bronze : un récit pétersbourgeois (1833), partie II, 1. 188, où la statue animée du tsar Pierre galope avec une réverbération pesante (je donne à nouveau la valeur du o selon sa position)

pa patryasyônnoy mastovôy.

sur le pavé tremblant.

Voici les deux rapides $p a$ 's identiques, faisant exploser la ligne, la rendant encore plus sonore (en correspondant au rythme des sabots de bronze). Certaines rues de St. Petersburg étaient pavées, d'autres macadamisées. Des revêtements consistant en des blocs hexagonaux de bois de pin taillés comme l'œuvre d'un menuisier furent introduits vers $1830 .{ }^{46}$

en soi-même l'unique objectif de sa conduite et, comprenant qu'il est l'instrument d'une fin qui le dépasse, il s'apercevra qu'il sert à quelque chose. "

45 A. Pushkin, Eugene Onegin, A novel in verse, Translation by V. Nabokov, New York, Bollingen / Princeton University Press, 1964, p. 232 (t. II). Notre traduction.

46 A. Pushkin, Eugene Onegin, A novel in verse, op. cit., p. 166 (t. I). Notre traduction. 
De manière singulière, Nabokov s'intéresse à la matière des pavés et grossit le détail. L'image confronte la tendresse du bois au métal des sabots qui produisent un bruit sourd en laissant leur empreinte. Leur dureté fait trembler la ville comme si elle était douée de vie, en même temps que la sensation marque la mémoire du lecteur : c'est bien d'un ébranlement de son imagination qu'il s'agit, qui rappelle que "l'œuvre [...] ouvre à l'expérience du monde ${ }^{47}$. En se saisissant d'un détail, la note du commentaire de Nabokov vise donc une universalisation par une relation dialectique entre la sensation et les mots.

L'écrivain reprendra cette image dans son œuvre intitulée La course du fou (qu'il traduit en anglais en 1964) et l'associera au passage d'un fiacre tout droit sorti de romans réalistes français ${ }^{48}$ pour marquer l'envahissement du détail chez un autre petit : l'enfant-champion d'échecs, Loujine. Les échos qui existent entre les cours de littérature de Nabokov et ses traductions d'un côté, ses propres écrits de l'autre incitent à lire les deux parties de son ouvre de manière conjointe ${ }^{49}$. Dans la scène où le pavé de bois réapparaît, ce dernier espionne un ami de son père manipulant ce que l'on soupçonne être une pièce de bois (on aimerait penser qu'il s'agit d'un cavalier, mais le texte ne précise pas), avant que l'enfant ne soit pour la première fois mis en contact avec l'objet de sa passion : le jeu qui altèrera toute sa vie.

Dissimulé dans l'ombre, Loujine le regardait sans oser bouger, stupéfait de voir un étranger s'installer confortablement à la table de son père. « Non, j’ai fini de jouer ", dit-il, les yeux au plafond, tandis que de sa main blanche et nerveuse il tripotait un objet sur la table. On entendit le bruit assourdi d'un fiacre roulant sur le pavé de bois. ${ }^{50}$

Au-delà de la particularité des situations, ce que cherche Nabokov relève d'un partage du sensible aux multiples effets, dont le plus évident serait le refus de l'indifférence du lecteur. L'auteur vise à dépasser la communauté nationale en proposant une description des matières.

Le grossissement du détail invite à réfléchir aux représentations du monde, alors que l'on propose dans le même temps une réflexion sur les dangers des analogies. En associant la langue russe à un imaginaire français, Nabokov trouve une brèche dans la nuit européenne, car comme l'explique Pascale Casanova, " la reconnaissance critique en français de La course du fou permet de sortir [...] des limites "nationales" de la communauté russe en exil et lui permet

47 Antoine Berman, La Traduction et la lettre, ou l'auberge du lointain, Paris, Éditions du seuil, 1999 , p. 70.

48 On peut penser à Madame Bovary de Flaubert ou à La Curée de Zola.

49 Voir Isabelle Poulin, Discours littéraire et discours didactique : Vladimir Nabokov, professeur de littératures, thèse de doctorat, [s.n.], 1993.

50 V. Nabokov, La Défense Loujine, traduit par Genia et René Cannac, révisé par Bernard Kreise, dans Euvres romanesques complètes I, édité par Maurice Couturier (dir.), Paris, Éditions Gallimard, 1999, p. 373. 
d'échapper à l'anathème de la critique russe, assez hostile à son livre. [...] En l'espace d'une semaine, et avant même que le roman n'ait été entièrement publié en russe, Nabokov signe un contrat avec Fayard pour la traduction française ${ }^{51}$. L'insertion de l'image pouchkinienne dans une autre œuvre permet d'enrichir le fonds culturel de la République des Lettres. La référence à Pouchkine échappe à la vue du lecteur pressé, mais sur le temps long, l'hybride nabokovien permet de prendre conscience de la fécondité de l'œuvre russe souvent mésestimée. Elle acquiert enfin un sens politique qui n'est pas sans conséquences sociales. «Avec l'avènement de la littérature mondiale, [...] les diverses cultures cherchent désormais à se contempler dans le miroir des autres, à y chercher ce qu'elles ne peuvent pas percevoir d'elles-mêmes. La saisie de soi ne passe plus seulement par la saisie de l'étranger, mais par celle que l'étranger a de nous. " ${ }^{52}$

En 1961, Landolfi traduit le même passage par : «Per lo squassato lastrico », où apparaissent lastrico, le "pavé ", et le participe passé de squassare, squassato, "trembler violemment", ce qui permet au traducteur de conserver l'homophonie initiale en $[s],[t],[a],[o]$. Plus que la syntaxe qu'il modifie, Landolfi tente ainsi de donner à entendre le rapport très étroit du texte premier à sa matière sonore à travers le staccato du pas du cheval. Un autre passage du poème de Pouchkine - les vers 107 à 109 -, dans le commentaire duquel Nabokov rattache le nom du protagoniste à un geste de réécriture, montre le soin pris par Landolfi pour trouver des équivalents.

\section{Traduction de Landolfi}

Il Cavaliere del bronzo (1961)

98 Sull'oscurata Pietrogrado

Freddo d'autunno novembre ventava.

100 Risciacquando con onda fragorosa

Ai margini della ben fatta cinta,

La Neva s'agitava come infermo

Smanioso nel suo letto.

Era già tardi e scuro;

105 Alla finestra batteva stizzosamente la pioggia,

Soffiava il vento, tristemente urlando.

Da una visita, a casa, in quella,

Il giovane Eugenio tornava...

Il nostro eroe

110 Chiameremo con questo nome. Il quale

Suona gradito; e con esso da tempo

112 È la mia penna inoltre familiare.

51 P. Casanova, La République mondiale des Lettres, op. cit., p. 206.

52 Antoine Berman, L'Épreuve de l'étranger. Culture et traduction dans l'Allemagne romantique, Paris, Gallimard, 1984, p. 104. 


\section{Traduction de Nabokov}

The Bronze horseman (1964)

98 O'er the ensombered Town of Peter

November breathed with autumn chill.

100 Plashing with noisy wave against

the margins of her trim embankment, the Neva tossed about like a sick man upon his restless bed. 'Twas late and dark. The rain

105 beat crossly on the windowpane, and the wind blew with a sad howl.

At this time from a visit came home young Eugene... We'll call our hero

110 by this name. It sounds pleasingly. With it, moreover,

112 my pen somehow has long been friends.

Texte de Pouchkine

Медный всадник $(1833)^{53}$

98 Над омрачённым Петроградом Дышал ноябрь осенним хладом.

100 Плеская шумною волной В края своей ограды стройной, Нева металась, как больной В своей постеле беспокойной. Уж было поздно и темно;

105 Сердито бился дождь в окно, И ветер дул, печально воя.

В то время из гостей домой Пришёл Евгений молодой... Мы будем нашего героя

110 Звать этим именем. Оно Звучит приятно; с ним давно

112 Моё перо к тому же дружно.

Landolfi tente de restituer l'équilibre sonore du vers. Nous pouvons nous en rendre compte au vers 108 où les sonorités en [1] et en [oj] dans пришёл et молодой se retrouvent dans le texte italien, sous une autre forme, dans giovane et Eugenio où revient le groupe sonore [jo]. La traduction vise le passage d'images à partir d'une étude de la lettre. Ainsi tout en étant très proche du texte russe, on espère partager avec des nonrussophones la virtuosité d'une construction poétique. Autre procédé dans Des mois : en février 1964, le personnage interroge, à l'occasion d'une évocation du déluge, la misère de l'homme, perçue à travers le prisme d'une goutte d'eau qui diffracte et rappelle un monde disparu.

53 C'est nous qui soulignons. 
La pluie était un défi et un tourbillon; comme dans certains mythes où les héros tombent du ciel, chaque goutte en frappant le sol se transformait en chevalier armé ; des nappes d'eau montait un appel ; l'air, mystérieusement renouvelé, me cornait aux oreilles : "Viens. Tu disais que tu voulais faire quelque chose, c'est le moment. Et pas seulement quelque chose, tu peux tout faire. » $\mathrm{Ou}$ le vent : il arrivait comme un fauve, regardait autour de lui, haletant, certain que ceux qui possédaient encore leurs ailes originelles le suivraient. Mais je n'étais pas capable, même à ce moment-là de répondre à leur appel : non par lâcheté, mais par méfiance, ce qui est pire. Et, ô merveilleuses apparences de la pluie calmée, du vent replié, tombé ! Dans tout cela il y avait de l'étonnement, de la stupeur, plus que des reproches ou du mépris ; cependant, quoi de plus amer que ces mots, lus dans les éléments déçus : "Tu n’es pas digne d'être sauvé » ?54

Le lecteur n'est pas oublié dans cette parodie de voix angoissées qui emprunte à la Bible comme au Cavalier de bronze. Il est pris à témoin voire requis par l'usage de la deuxième personne. Si le texte ne le pousse pas à agir, il offre, par son étrangeté, une réflexion sur les signes et leur pouvoir de sollicitation.

Rendre le staccato du pas du cheval ou le langage familier d'Eugène relève de choix, car en se concentrant sur ces éléments, Landolfi et Nabokov en oublient beaucoup d'autres puisqu'il leur était impossible de rendre compte de toutes les dimensions sonores, visuelles, intertextuelles, prosodiques entre autres, du texte russe. Leurs traductions s'adressent avant tout à un lecteur individuel qu'elles engagent d'une autre manière, à travers un partage du sensible. L'objectif de ces deux écrivains-traducteurs est de rendre perceptible une construction esthétique en refusant de faire des mots de simples vecteurs du sens. Landolfi et Nabokov touchent les affects du lecteur. Sans exclure la mise en place d'un lien social, ils font de la littérature une expérience sensible, unique dans son appréhension du monde.

\section{Conclusion}

Pouchkine est devenu le poète national russe au prix d'une construction élaborée sous l'Empire, confortée par le stalinisme. Certes les images de la réalité russe et la simplicité du poète parlaient à un grand nombre de ses contemporains, mais c'est la reconnaissance à large échelle du caractère russe de son œuvre qui a permis d'établir un lien social. Une telle élaboration met la littérature au service de la société. Pour contourner ces représentations qui oublient le texte, Vladimir Nabokov, "le plus "mondial" des écrivains russes de l'émigration " ${ }^{55}$, choisit de prélever un emblème dans Le Cavalier de bronze et d'insister sur le matériau verbal qui le compose. Landolfi cherche pour sa part à repousser les limites de l'espace littéraire italien, pétrifié dans le face-àface avec les grands poètes du XIV siècle.

54 T. Landolfi, Des mois, op. cit., p. 103.

55 Wladimir Troubetzkoy, «La Russie et l'Europe. Mille ans d'histoire », in Russies Poccuu. Mélanges offerts à Georges Nivat pour son soixantième anniversaire, Lausanne, Éditions L'Âge d'homme, 1995 , p. 62. 
Si l'on ne veut pas forcer le lien entre les peuples, le lien social russe semble difficile à percevoir, car comme l'écrit Georges Nivat, à propos de la France, « la traduction évolue avant tout dans un terreau d'attente : les platesbandes sont prêtes pour telle ou telle culture, gare à la plante inattendue qui s'y hasarderait! Pouchkine est dans nos "lettres russes" le grand perdant du passage culturel : il n'existe pas dans l'imaginaire français " ${ }^{56}$.

En ambitionnant une littérature-monde à très petite échelle, Landolfi et Nabokov souhaitent un lecteur éclairé et autonome puisqu'ils soulignent le caractère ouvert et déconcertant du texte de Pouchkine. Ils refusent d'orienter la lecture et donnent aux mots la prééminence en espérant une "intertraduction $[\ldots]$ généralisée $"{ }^{57}$. Le "dissensus " et le malentendu demeurent possibles, mais la responsabilité de chacun se trouve engagée. Demander d'apprendre le russe pour connaître le texte de Pouchkine n'est pas, dans ces conditions, une ultime provocation. Cette position se fonde sur une éthique qui réclame du lecteur un vrai engagement vers l'autre en même temps qu'une réflexion lucide sur les frontières. Le lien ainsi mis en place entre un texte et une communauté de lecteurs n'est pas uniquement intersubjectif. Il passe par des tensions entre une langue, des valeurs et la richesse d'une expérience intérieure. Ainsi perçue, la traduction envisage les identités collectives sur un mode plus lent, mais moins violent. Le lien social ne lui préexiste pas. Il apparaît, virtuel, à l'horizon.

\title{
Stanislas Gauthier
} EA 4195 TELEM

Université Michel de Montaigne Bordeaux 3 Stanislas.Gauthier@etu.u-bordeaux3.fr

\begin{abstract}
Résumé
Landolfi et Nabokov traduisent pendant la Guerre froide le Cavalier de bronze, un long poème narratif de Pouchkine qui devint progressivement le chef-d'œuvre national russe. Contrairement aux autres traducteurs qui traduisent d'une manière globale, ces deux écrivainstraducteurs travaillent à très petite échelle. Visant la restitution d'affects, ils commentent en des micro-récits les mots de Pouchkine et laissent virtuelle la possibilité de fonder des liens sociaux internationaux.
\end{abstract}

\section{Mots-clés}

Pouchkine, Nabokov, Landolfi, sociologie de la littérature, études de traduction.

56 Georges Nivat, "La traduction, passage non obligé... ", in La Quinzaine littéraire, n 613, du $1^{\text {er }}$ au 15 décembre 1992, p. 12-13.

57 A. Berman, L'Épreuve de l'étranger. Culture et traduction dans l'Allemagne romantique, op. cit., p. 94. 


\begin{abstract}
During the Cold War, Landolf and Nabokov translate The Bronze Horseman, a long narrative poem by Pushkin that became the Russian national masterpiece. Unlike to the other translators of their time who translate as a whole, those two writers and translators work at a very small scale. Searching a new experience of affects, they comment the words of Pushkin through micro-tales, and let virtual the possibility to create international social bonds.
\end{abstract}

Keywords

Pushkin, Nabokov, Landolf, social function of literature, translation studies.

\title{
BIBLIOGRAPHIE
}

André Akoun et Pierre Ansart, Dictionnaire de sociologie, Paris, Robert-Le Seuil, 1999.

Louis Aragon, Chroniques de la pluie et du beau temps, précédé de Chroniques du Bel Canto. Paris, Les Éditeurs Français Réunis, 1979.

Antoine Berman, L'Épreuve de l'étranger. Culture et traduction dans l'Allemagne romantique. Paris, Gallimard, 1984.

—, La Traduction et la lettre, ou l'auberge du lointain. Paris, Éditions du Seuil, 1999.

Pascale Casanova, La République mondiale des Lettres, Paris, Éditions du Seuil, 2008.

Michele Colucci, "Le traduzioni italiane del Novecento di poesia puškiniana ", in Paola Buoncristiano, Puškin, la sua epoca e l'Italia, Soveria Mannelli, Rubbettino, 2001.

Paul Debreczeny, Social Functions of Literature. Alexander Pushkin and Russian Culture, Stanford, Stanford University Press, 1997.

Jacques Derrida, "Qu'est-ce qu'une traduction relevante? ", in Quinzièmes Assises de la traduction littéraire, Arles, Actes Sud, 1999.

Émile Durkheim, le Suicide. Étude de sociologie, Paris, PUF, 1930.

Florence Goyet, «L'Épopée » [en ligne] : http://www.vox-poetica.com/sflgc/biblio/goyet.html (page consultée le 28 mars 2013).

Julien Gracq, La littérature à l'estomac, Paris, José Corti, 1950.

Roman Jakobson, Russie, folie, poésie, Paris, Seuil, 1986.

Clyde Kluckhohn, "Myths and Rituals: A General Theory », in The Harvard Theological Review, vol. $35, \mathrm{n}^{\circ} 1,1942$.

Tommaso Landolfi et Idolina Landolfi, Opere I, 1937-1959, Milan, Rizzoli, 1991.

Tommaso Landolfi, Des Mois, traduction de l'italien de Monique Baccelli, Paris, Éditions Allia, 1995.

_-, "Un antico canto epico », in Gogol' a Roma, Milan, Adelphi Edizioni, 2002.

Lauren G. Leighton, A Bibliography of Alexander Pushkin in English. Studies and Translations, Lewiston, The Edwin Mellen Press, Studies, in Slavic Languages and Literature, vol. 12, 1999.

Catherine Lemagnen, "Le concept de narodnost' dans la pensée russe du XIX siècle ", in "Monde russe et identités. " La Revue russe 36, Paris, Institut des études slaves, 2011, p. 11 à 20 .

Mikhail Lermontov, Euvres poétiques, Lausanne, L’Âge d'homme, 1985.

Gaston Mialeret et Jean Vial, Histoire mondiale de l'éducation, t. 3 de 1815 à 1945, Paris, PUF, 1981. 
Vladimir Nabokov, Edmund Wilson Correspondance 1940/1971. Traduit de l'anglais par Christine Raguet-Bouvard, Paris, Rivages, 1979.

Vladimir Nabokov, Song of Igor's Campaign, New York, Ardis, 1960 (1988 pour l'édition consultée).

—, Lettres choisies 1940-1977. Traduction de l'anglais de Christine Bouvart, Paris, Gallimard, 1989.

—- La Défense Loujine, traduit par Genia et René Cannac, révisé par Bernard Kreise, dans Euvres romanesques complètes I, édité par Maurice Couturier (dir.), Paris, Éditions Gallimard, 1999.

Georges Nivat, "Un styliste de l'histoire", in Vers la fin du mythe russe. Essais sur la culture russe de Gogol à nos jours, Lausanne, L'Âge d'homme, 1983.

—, "La traduction, passage non obligé... ", in La Quinzaine littéraire, n 613, du $1^{\text {er }}$ au 15 décembre 1992.

—_, "La Treizième tribu ", in Russie-Europe. La Fin du schisme. Études littéraires et politiques, Lausanne, L'Âge d'homme, 1993.

Georges Nivat (éd.), Transitions. "Bilan de la culture soviétique ", vol. XLI, Bruxelles, Critème/Ieug, 2002.

Alexandre Pouchkine, Le Cavalier de bronze, traduit sur les manuscrits originaux et publié par André Meynieux, Paris, Éditions André Bonne, 1959.

Aleksandr Puškin, Poemi e liriche, versioni, introduzione e note di Tommaso Landolfi, Torino, Einaudi, 1961.

Aleksander Pushkin, Eugene Onegin, A novel in verse, translation by Vladimir Nabokov, New York, Bollingen / Princeton University Press, 1964.

Александр Пушкин, Полное собрание сочинений в одном томе, Moscou, Издательство Альфа Книга, 2010.

Isabelle Poulin, Discours littéraire et discours didactique : Vladimir Nabokov, professeur de littératures, thèse de doctorat, [s.n.], 1993.

Claude Quétel (dir.), Dictionnaire de la Guerre froide, Paris, Larousse, 2008.

Martin Rueff, «Entretien avec Jean-Patrice Courtois », Le nouveau recueil, n 81, Paris, Champ Vallon, décembre 2006-février 2007.

Christopher Rundle, "Fascist Italy ", in Derek Jones, Censorship : a world encyclopedia. Londres, Fitzroy Dearborn, 2001

Philippe Sellier, "Qu'est-ce qu'un mythe littéraire ? ", in Littérature, vol. 55, n 55, p.112-126.

Claudia Scandura, Letteratura russa in Italia. Un secolo di traduzioni, Rome, Bulzoni Editore, 2001.

André Siniavski, Promenades avec Pouchkine, traduit du russe par Louis Martinez, Paris, Éditions du Seuil, 1975.

Ferdinand Tönnies, Communauté et Société, Paris, PUF, 2010.

Wladimir Troubetzkoy, "La Russie et l'Europe. Mille ans d'histoire ", in Russies России Mélanges offerts à Georges Nivat pour son soixantième anniversaire, Lausanne, Éditions L'Âge d'homme, 1995.

Boris A. Uspenskij, Storia della lingua letteraria russa. Dall'antica Rus' a Puskin. Edizione a cura di Nicoletta Marcialis, Roma, Il Mulino, 1993.

Stéphane Vibert, "La quête russe de l'universel : mouvement slavophile et hiérarchie de valeurs socio-communautaires (1825-1855) ", in Revue des études slaves, t. 73, fascicule 2-3, 2001, p. 519-530. 